\title{
Impact of Outdoor Play in Sunlight - An Additive Factor with Atropine 0.01\% for Preventing Myopia Progression
}

\section{Kaushik Murali ${ }^{*}$, Vidhya $\mathrm{C}^{2}$ and Anshupa ${ }^{3}$}

${ }^{1}$ Department of Pediatric Ophthalmology and Strabismus, President, Medical

Administration, Sankara Eye Hospitals, India

${ }^{2}$ Consultant, Department of Pediatric Ophthalmology and Strabismus, Sankara Eye

Hospital, Bangalore, India

${ }^{3}$ DNB Fellow, Sankara Eye Hospital, Bangalore, India

*Corresponding Author: Kaushik Murali, Department of Pediatric Ophthalmology

and Strabismus, President, Medical Administration, Sankara Eye Hospitals, India.
Received: January 27, 2020

Published: February 27, 2020

(C) All rights are reserved by Kaushik

Murali., et al.

DOI: $10.31080 /$ ASOP.2020.03.0098

\begin{abstract}
Aim: The aim is to evaluate the additive role of outdoor play along with Atropine $0.01 \%$ in further controlling the progression of myopia in Indian children.

Methods: Children with progressive myopia were started on atropine $0.01 \%$ along with outdoor play. Children were divided into those who played outdoors for $<1$ hour, 1 - 2 hours and $>2$ hours per day. Effect of outdoor activity on progression in spherical power of myopia was correlated.

Results: 55 children with progressive myopia were included. $50.9 \%$ of children spent less than 1 hour daily in sunlight, $12.7 \%$ spent more than 2 hours outdoors. Myopia progression of $0.12 \mathrm{D}$ was noted in children played $>2$ hours per day when compared to those who played less than one hour (0.87D). Those with family history showed a slower progression with increased outdoor play - 0.33D/ year compared to - $0.68 \mathrm{D} /$ year in children who played less than 2 hours a day in sunlight.

Conclusion: Outdoor play has an additive effect along with atropine $0.01 \%$ in controlling the progression of myopia.

Keywords: Progressive Myopia; Outdoor Play; Atropine 0.01\%
\end{abstract}

\section{Introduction}

High myopia is a public health and economic challenge due to devastating visual prognosis associated with complications such as glaucoma, myopic retinopathy, and retinal detachment. Therefore, it is imperative to develop effective interventions to prevent myopia onset and progression as much as possible. Genetic and environmental factors have been believed to play an important role in the determination of refractive error [1]. Atropine $0.01 \%$ has been demonstrated to be safe and effective in controlling myopia progression $[2,5,6]$.

Several observational studies have been done to prove the hypothesis that outdoor play is associated with prevention of occurrence/ progression of myopia [4,8,9,11].

With the hypothesis that increasing time spent outdoors may be beneficial for the visual health of children, we tried to evalu- ate the additive role of outdoor play along with Atropine $0.01 \%$ in further controlling the progression of myopia in Indian children.

\section{Materials and Methods}

Children with progressive myopia where progression is defined as increase in spherical equivalent by $>0.75 \mathrm{D}$ within 6 months period, were started on atropine eye drops. Atropine $0.01 \%$ eye drops were given for application to both eyes, one drop at bed time for a period of one year. Those with the baseline myopia of $>2 \mathrm{D}$, in the age group of 3 - 15 years with progressive myopia were included in the study.

The children were specifically encouraged to increase their time spent outdoors in playing using visual posters to educate parents and through a detailed interaction with the authors.

Children with developmental delay, ocular media opacities, retinal dystrophies, ocular trauma/surgery and amblyopia were 
excluded. Every six months, parents were given a questionnaire regarding the time spent outdoors by their children. Children were also asked about their daily schedule to confirm the data shared in the questionnaire. If there found to be a discrepancy, the candidate was excluded from the study. Details of myopia in the parents were also noted.

Any adverse effects of atropine $0.01 \%$ eye drops (in the form of photophobia, eye allergies) were documented.

Children were further divided into those who played outdoors for $<1$ hour, 1 - 2 hours and $>2$ hours per day. Effect of outdoor activity on progression in spherical power was correlated.

The data was entered in Microsoft excel and then transferred to SPSS version 20.0. Descriptive statistics was done for all the variables. The association was done by chi square test. Progression was seen by students paired t test. Visit wise comparison was done by ANOVA with Bonferroni t test. Correlation and regression was also done. A non-parametric test Mann-Whitney U test was done where the data is skewed. $\mathrm{P}<0.05$ was found to be statistically significant.

\section{Results}

Eyes of 55 children were enrolled for this study and their data analyzed. $58.1 \%$ of the children were males. The mean age of children being $10.27+2.8$ years (range $-4-15$ years). The spherical power of myopia ranged from $2-10 \mathrm{D}$, the mean being 4.4 D.

After starting the children on treatment, 20 children out of 55 showed no progression of myopia over a period of one year. 1 child showed a decrease in the spherical power by $0.5 \mathrm{D}$ over a period of one year. 34 children showed progression in their spherical power out of which 9 had a progression of at least $0.75 \mathrm{D} /$ year, however they showed a reduction in progression of nearly $50 \%$ compared to the average prior to increase in drops. Out of the 34 children, there were 3 children who continued to progress $>1.5 \mathrm{D}$ after treatment. Even these had an $84 \%$ reduction in progression when compared to progression before starting the drops.

$50.9 \%$ of children spent less than 1 hour daily in sunlight, $12.7 \%$ spent more than 2 hours outdoors, the maximum time spent was 3.2 hours.

It was observed that the children who played outdoor for $>2$ hours per day had minimal progression of spherical power, both at the end of 6 months and one year, which showed statistical significance ( $p-0.01)$. The progression is more in children with $<1$ hour of outdoor activity.

\begin{tabular}{|l|c|c|c|}
\hline \multicolumn{1}{|c|}{ Outdoor Play } & $<\mathbf{1}$ Hour & 1-2 Hours & $>\mathbf{2}$ Hours \\
\hline Mean age of children (years) & 9.8 & 9.9 & 10.1 \\
\hline No. of children & 28 & 20 & 7 \\
\hline Mean spherical power (Dioptres) & $4.68 \mathrm{D}$ & $4.15 \mathrm{D}$ & $4.2 \mathrm{D}$ \\
\hline
\end{tabular}

Table 1: Showing the distribution in various categories of outdoor play.

\begin{tabular}{|l|c|}
\hline \multicolumn{1}{|c|}{ Outdoor Play } & Myopia Progression In 1 Year \\
\hline$<1$ hour & $0.87 \mathrm{D}$ \\
\hline $1-2$ hours & $0.35 \mathrm{D}$ \\
\hline$>2$ hours & $0.12 \mathrm{D}$ \\
\hline
\end{tabular}

Table 2: Outdoor play and myopia progression.

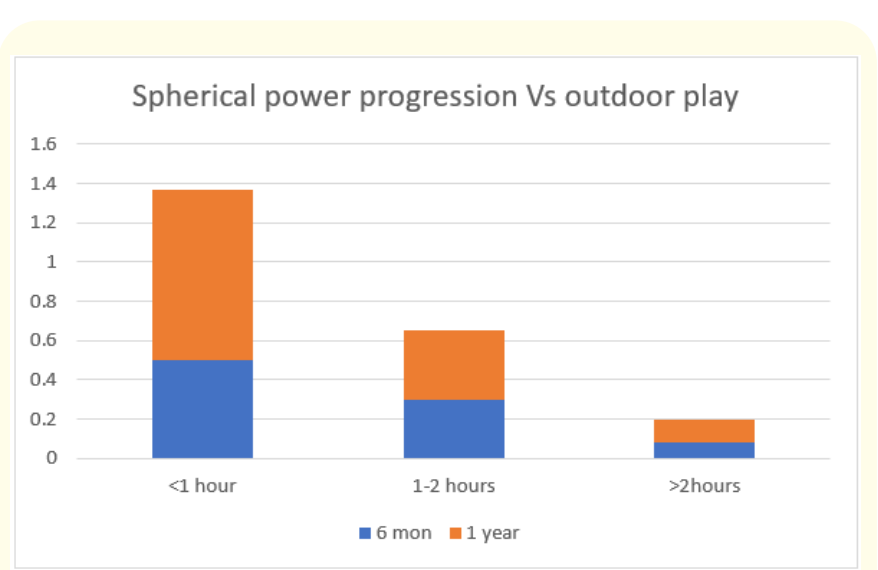

Figure 1: Showing the spherical power progression with respect to outdoor play in 6 months and at the end of one year.

Maximum progression noted in this cohort was of - 2.25D. This child was noted to have a family history of myopia in both parents and also had minimal outdoor play of less than 1 hour every day.

46 out of 55 children had myopic parents.

\begin{tabular}{|l|c|c|}
\hline \multicolumn{2}{|c|}{$\begin{array}{c}\text { Annual Spherical Power Progression } \\
\text { Children Playing Outdoors < 2 Hrs }\end{array}$} & $\begin{array}{c}\text { Playing Outdoors } \\
\text { > 2hrs }\end{array}$ \\
\hline Myopic parents & $-0.68 \mathrm{D}$ & $-0.33 \mathrm{D}$ \\
\hline Without myopic parents & $-0.75 \mathrm{D}$ & 0 \\
\hline
\end{tabular}

Table 3: Spherical progression in children with outdoor activity.

We calculated the time of play outdoors from the interview with the children on their previous days schedule and found it to correlate with the questionnaires.

No adverse effects of the atropine was noted. 


\section{Discussion}

Numerous research studies have been undertaken to understand the causative mechanisms for Myopia and hence the treatment modalities in controlling the progression of myopia. There have been studies that have demonstrated effectiveness of $0.01 \%$ atropine among Indian children $[2,5,6]$.

The effect of Atropine towards prevention of myopia progression seems to be multifactorial. There have been some research to show the possible need to increase the concentration of atropine in Indian population [7]. This study is unique in literature to analyze the role of outdoor play as an additive effect in the control of myopia progression.

Various studies have been demonstrated to show the beneficial effects of outdoor play in the control of myopia progression. The school-based Guangzhou Outdoor Activity Longitudinal (GOAL) Study on children aged 6 to 7 years found that children who had one extra hour of structured outdoor activities added to the school day had statistically significant reductions in axial elongation after 1 year [3]. Lisa Jones., et al. in their 11 year comparative study observed that children who developed myopia, stayed outdoors 4 hours lesser as compared to the ones who remained non myopic who stayed outdoors $11.65+6.97$ hours per week [4]. Rose., et al. in an Australian study found that children with increased near work and low outdoor time were 2 - 3 times more likely to be myopic compared to those performing low near work and increased outdoor activities [8].

Dirani., et al. found a significant negative association between progression of myopia and outdoor time spent. For each hour increase in outdoor activity, the spherical error decreased by $0.17 \mathrm{D}$ and axial length decreased by $0.06 \mathrm{~mm}$ [9].

The results of our study showed that the outdoor play in sunlight for more than 2 hours per day, seemed to have an additive effect along with atropine $0.01 \%$ on controlling the progression of Myopia. None of the children had any photophobia or any other adverse effects while playing outdoor in the sunlight. This proves that atropine $0.01 \%$ eye drops are safer in Indian climatic conditions.

Although an association between myopia prevalence and time spent outdoors was relatively consistent among several studies, the underlying mechanism of protection against myopia onset and progression was less clear. Bright light outdoors may be the most possible mechanism. Brighter light potentially reduces the development of myopia by pupil constriction, resulting in less visual blur, or by stimulation of dopamine release from the retina. Dopamine has been known to be an inhibitor of axial elongation [10]. Higher light intensity outdoors could make the depth of field greater and decrease image blur. Spectral composition of light rather than intensity seems to be a more important factor [11].

46 out of 55 children had myopic parents. Since the sample size is small we are unable to comment on the correlation. The SMS [12] reported that children with one or both parents myopic had to two and eight times higher risk of developing myopia compared with children who had no myopic parents. While the rates of progression showed no direct correlation with family history, those with family history showed a slower progression with increased outdoor play - $0.33 \mathrm{D} /$ year compared to $-0.68 \mathrm{D} /$ year in children who played less than 2 hours a day in sunlight.

Our study was however limited on the reporting of the time of daily play by the parent. The children in the group of outdoor play of $>2$ hours were very less to draw a firm conclusion on the effect of increased time outdoors as a linear impact. Further randomized controlled trials are required to prove this association.

The effect of Atropine towards prevention of myopia progression seems to be multifactorial. The outdoor play has definitely a role to play with atropine in controlling the progression.

\section{Conclusion}

Outdoor play has an additive effect along with atropine $0.01 \%$ in controlling the progression of myopia. Even in children with positive family history of myopia were found to have reduced progression with outdoor play. So children should be encouraged to play outdoors in sunlight for at least 2 hours a day to prevent the progression.

\section{Bibliography}

1. You QS., et al. "Prevalence of myopia in school children in greater Beijing: the Beijing childhood Eye study". Acta Ophthalmologica 92.5 (2014): 398-406.

2. Vidhya., et al. "Safety and efficacy of reconstituted atropine 0.01\% eye drops for Indian children with myopic progression". Asian Journal of Ophthalmology (accepted for publication).

3. Morgan IG., et al. "Twoyear results from the Guangzhou outdoor activity longitudinal study (GOALS)". ARVO Meet Abstracts 53 (2012): 27-35. 
4. Jones LA., et al. "Parental history of myopia, sports and outdoor activities, and future myopia". Investigative Ophthalmology and Visual Science 48 (2007): 3524-3532.

5. Chia A., et al. "Five-Year Clinical Trial on Atropine for the Treatment of Myopia 2: Myopia Control with Atropine 0.01\% Eyedrops". Ophthalmology 123 (2016): 391-399.

6. Jason Yam JC., et al. "Low-concentration atropine for myopia progression (LAMP) study: a randomized, double-blinded, placebo-controlled trial of $0.05 \%, 0.025 \%$, and $0.01 \%$ atropine eye drops in myopia control". Ophthalmology 126 (2019): 113-124.

7. Mihir Kothari and Vivek Rathod. "Efficacy of $1 \%$ atropine eye drops in retarding progressive axial myopia in Indian eyes". Indian Journal of Ophthalmology 65 (2017): 1178-1181.

8. Rose KA., et al. "Outdoor activity reduces the prevalence of myopia in children”. Ophthalmology 115 (2008): 1279-1285.

9. Dirani M., et al. "Outdoor activity and myopia in Singapore teenage children". British Journal of Ophthalmology 93 (2009): 997-1000.

10. Dong F., et al. "Inhibition of experimental myopia by a dopamine agonist: different effectiveness between form deprivation and hyperopic defocus in guinea pigs". Molecular Vision 17 (2011): 2824-2834.

11. Mehdizadeh $\mathrm{M}$ and Nowroozzadeh MH. "Outdoor activity and myopia”. Ophthalmology 116 (2009): 1229-1230.

12. Ojaimi E., et al. "Methods for a population-based study of myopia and other eye conditions in school children: the Sydney Myopia Study". Ophthalmic Epidemiology 12.1 (2005): 59-69.

\section{Assets from publication with us}

- Prompt Acknowledgement after receiving the article

- Thorough Double blinded peer review

- Rapid Publication

- Issue of Publication Certificate

- High visibility of your Published work

Website: www.actascientific.com/

Submit Article: www.actascientific.com/submission.php

Email us: editor@actascientific.com

Contact us: +919182824667

Citation: Kaushik Murali., et al. "Impact of Outdoor Play in Sunlight - An Additive Factor with Atropine 0.01\% for Preventing Myopia Progression". Acta Scientific Ophthalmology 3.3 (2020): 21-24. 